\title{
Endoscopic-Assisted Middle Fossa Craniotomy for Resection of Vestibular Schwannoma
}

\author{
Brian S. Chen ${ }^{1}$ Daniel S. Roberts ${ }^{1}$ Gregory P. Lekovic ${ }^{1}$ \\ ${ }^{1}$ House Clinic, Los Angeles, California, United States \\ Address for correspondence Gregory P. Lekovic, MD, PhD, \\ J Neurol Surg Rep 2016;77:e1-e7. \\ Neurosurgical Associates of the House Clinic, 2100 West 3rd Street, \\ Suite 111, Los Angeles, CA 90057, United States \\ (e-mail: glekovic@hei.org).
}

\begin{abstract}
Keywords

- middle fossa craniotomy

- vestibular schwannoma

- endoscopic-assisted surgery

- hearing preservation

Background Rates of hearing preservation following surgery via middle fossa craniotomy in patients harboring tumors with unfavorable characteristics are significantly lower than for those patients with "favorable" tumors.

Objectives We will present two cases both with unfavorable conditions, which underwent endoscopic-assisted middle fossa craniotomy (MFC) resection of intracanalicular vestibular schwannomas with preserved postoperative hearing.

Methods Chart reviews were conducted on both patients. Their presentation, intraoperative details, and techniques, pre- and postoperative audiograms, and facial nerve outcomes are presented.

Results Patient A had $5.6 \times 6.8 \times 13.2 \mathrm{~mm}$ intracanalicular tumor with unserviceable hearing (pure tone audiometry [PTA], 41; speech determination score [SDS], 47\%; class D) but was blind so hearing preservation was attempted. Postoperative hearing was preserved (PTA, 47; SDS, 60\%; class B). Patient B had a $5 \mathrm{~mm}$ round intracanalicular tumor immediately adjacent to the vestibule and cochlea without any fundal fluid present. Preoperative audiogram showed serviceable hearing (PTA, 48; SDS, 88\%; class B). Postoperatively, aidable hearing was preserved (PTA, 51; SDS, 76\%; class C). Conclusion Hearing preservation surgery via MFC can be enhanced with endoscopicassisted dissection, especially in the lateral internal auditory canal. The superior optical view allows for preservation of cochlear nerve function and removal of residual tumor not otherwise seen on microscopy.
\end{abstract}

\section{Introduction}

In patients with serviceable hearing (i.e., American Academy of Otolaryngology-Head and Neck Surgery Hearing Classification [AAO-HNS] A or B) and an intracanalicular vestibular schwannoma (VS), tumor resection with preservation of hearing can be achieved via either middle cranial fossa (MFC) or retrosigmoid (RS) approaches. Although hearing preservation has been demonstrated for both approaches, hearing preservation for MFC (range, 20-71\%) has been shown in multiple studies to be higher than that obtained via the RS approach (range, 25-58\%). ${ }^{1-5}$ This higher rate of hearing preservation via middle fossa approach has been demonstrated from multiple groups at separate centers. However, the MFC approach is limited to smaller tumors ( $<$ $2.5 \mathrm{~cm}$ in our institution) because of comparatively less working room in the cerebellopontine angle (CPA), and as such is best employed for intracanalicular tumors, or those with limited CPA extension.

Importantly, a significant limitation of the either approach is visualization of intracanalicular tumors with lateral extension into the fundus of the internal auditory canal (IAC). This received

January 28, 2015

accepted after revision

August 10, 2015

published online

December 2, 2015
DOI http://dx.doi.org/

10.1055/s-0035-1564604. ISSN 2193-6358. (c) 2016 Georg Thieme Verlag KG
Stuttgart · New York

License terms

(®) $\Theta \circledast$ 
can be appreciated on preoperative thin cut axial T2-heavily weighted images; the value of this "fundal fluid" as a positive predictor of hearing preservation for MFC has been previously reported. ${ }^{5}$

The pathophysiological reason for poor hearing preservation with lateral VS resection at the fundus is unclear. We hypothesize that poorer surgical prognosis for hearing preservation is, at least in part, attributable to poor visualization of the fundus during surgery. Resection of tumor from the fundus under the operative microscope sometimes requires blind dissection of tumor. This is even more difficult when the tumor extends inferior to the transverse crest, which can sometimes measure up to $3 \mathrm{~mm}$ in length. ${ }^{6}$

We describe two patients with VS in which the endoscope was used to dissect tumor within the lateral fundus and remove residual tumor that was not visualized under microscopic dissection. Both patients were considered poor candidates for hearing preservation surgery, however, due to extenuating circumstances or personal preference, hearing preservation surgery was attempted. We discuss here the technique of endoscopic tumor dissection and surgical outcomes of both patients.

\section{Methods}

The charts of patients undergoing endoscopic middle fossa craniotomy for resection of acoustic neuroma were reviewed retrospectively. Patients were evaluated with regard to preand postoperative audiogram, contrast-enhanced magnetic resonance imaging (MRI), and facial nerve function (HouseBrackmann [HB] grade). Blood loss, length of procedure, and length of stay were obtained from the patient's inpatient chart. This study was conducted in accordance with institutional review board (IRB) policies (St. Vincent Medical Center IRB study \# 14-021).

\section{Case Histories}

\section{Patient A}

The patient is a 62-year-old male patient presenting with progressive right-sided hearing loss secondary to a small acoustic neuroma; he also complained of imbalance with several falls and veering to the right. The patient had a significant history of cryptococcal meningitis; sequelae of which included blindness (no light perception) bilaterally and shunt-dependent hydrocephalus. Preoperative audiogram showed a pure tone audiometry (PTA) of $41 \mathrm{~dB}$ and speech discrimination score (SDS) of $48 \%$ (AAO-HNS class D) in the right ear ( - Fig. 1). Preoperative contrast-enhanced MRI demonstrated a homogeneously enhancing mass of the right IAC $(5.6 \times 6.8 \times 13.2 \mathrm{~mm})$; T2-weighted constructive interference in steady state (CISS) imaging revealed a small amount of fundal fluid (-Fig. 2). The patient was advised as to the risks, benefits, and alternatives to surgery for this tumor. Specifically, he was initially felt to be a poor candidate for attempted hearing preservation because of his relatively poor preoperative hearing status and observation was recommended until the patient lost hearing or the tumor exhibited growth. Despite these recommendations, the patient elected for attempted hearing preservation surgery with the understanding that although serviceable hearing was not an expected outcome of surgery, even environmental noise awareness would be of value to him given the nature of his visual disability.

\section{Microsurgical Resection of Vestibular Schwannoma}

The patient underwent a standard right-sided middle fossa craniotomy for exposure of the IAC, as has been reported previously. ${ }^{7}$ After exposure of the posterior fossa dura and labyrinthine facial nerve, the dura was opened sharply and cerebrospinal fluid (CSF) drained from the exposed CPA. Fixed retraction/temporal lobe elevation was discontinued at this

\section{Case A Audiogram}

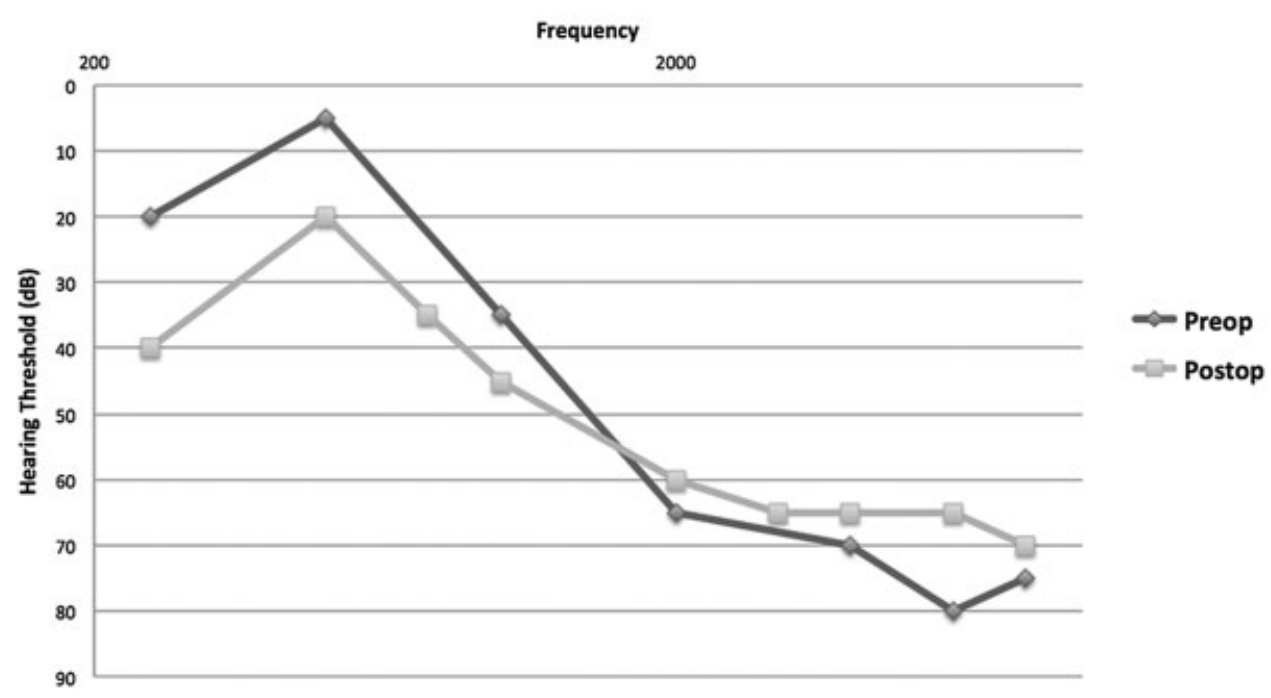

Fig. 1 Pre- and postoperative audiograms for patient A. Preoperative PTA is 41 (48\% SDS). Postoperative PTA is 47 (60\% SDS). PTA, pure tone audiometry; SDS, speech determination score. 


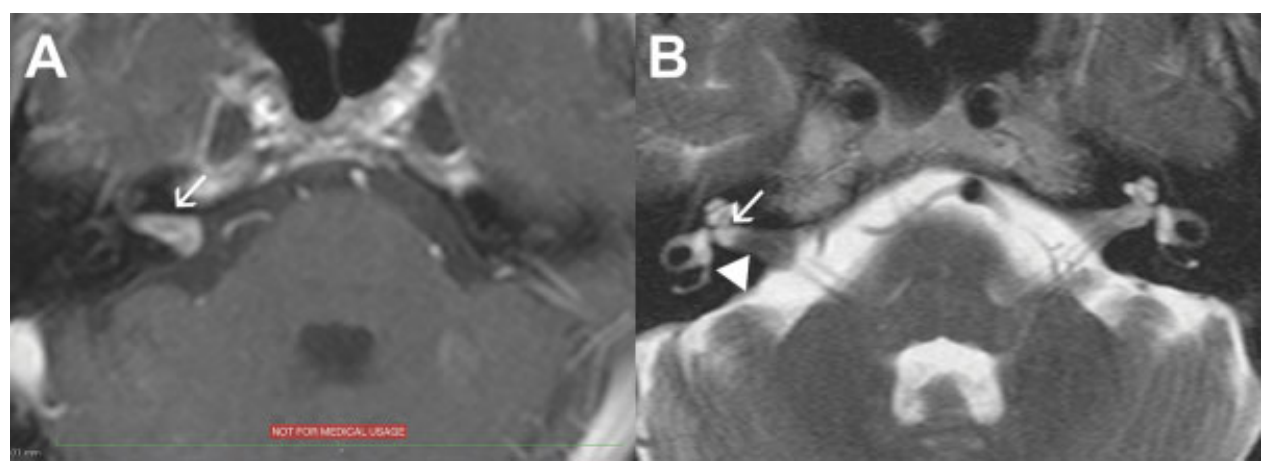

Fig. 2 Preoperative MRI for patient A. (A) T1-weighted MRI with contrast shows a right IAC lesion (white arrow) spanning from the porus to the lateral IAC. (B) T2-weighted MRI shows a filling defect (white arrowhead) in entire IAC with minimal fundal fluid (white arrow). IAC, internal auditory canal; MRI, magnetic resonance imaging.

point, and the VS was resected using standard microsurgical dissection techniques. At the completion of the microsurgical portion of the procedure, no tumor could be seen in the IAC, although the lateral extent could not be visualized directly (-Fig. 3A).

\section{Introduction of the Operative Endoscope}

After completion of the microsurgical resection to the maximal extent, the surgical endoscope was introduced into the field. The operative microscope focal length was lengthened to the maximal distance, allowing a $3-\mathrm{mm} 14-\mathrm{cm}$ Hopkins rod (Storz, Tuttlingen, Germany) to be introduced into the field under direct visualization. An endoscope with a 30-degree viewing angle was used. A microcottonoid was placed on the temporal lobe dura and flocculus of the cerebellum to protect these structures from heat-related injury, and the brain was cooled with intermittent irrigation. Upon introduction of the endoscope, residual tumor in the fundus of the IAC was immediately visible (-Fig. 3B). Using conventional microinstruments and microdissection techniques with visualization provide by the endoscope, this residual tumor was removed from the fundus of the IAC
(-Figs. 4 and 5; -Video S1, Supplementary Material available in the online version only).

\section{Supplemental Video 1 In this video, we present} patient A. The preoperative MRI shows the VS extending the entire length of the right IAC. After microscopic dissection, there appears to be no residual tumor. An endoscope is then brought in, under direct microscopic guidance, and reveals residual tumor in the fundus that is completely dissected with the endoscope. The video pans between the microscopic and endoscopic view, which illustrates the angles that cannot be seen with microscopy alone. After complete tumor removal, the fundus of the IAC to include the transverse crest can be inspected and no residual tumor is present. IAC, internal auditory canal; MRI, magnetic resonance imaging; VS, vestibular schwannoma. Online content including video sequence is available at: https://www.thieme-connect.com/ products/ejournals/html/10.1055/s-0035-1564604

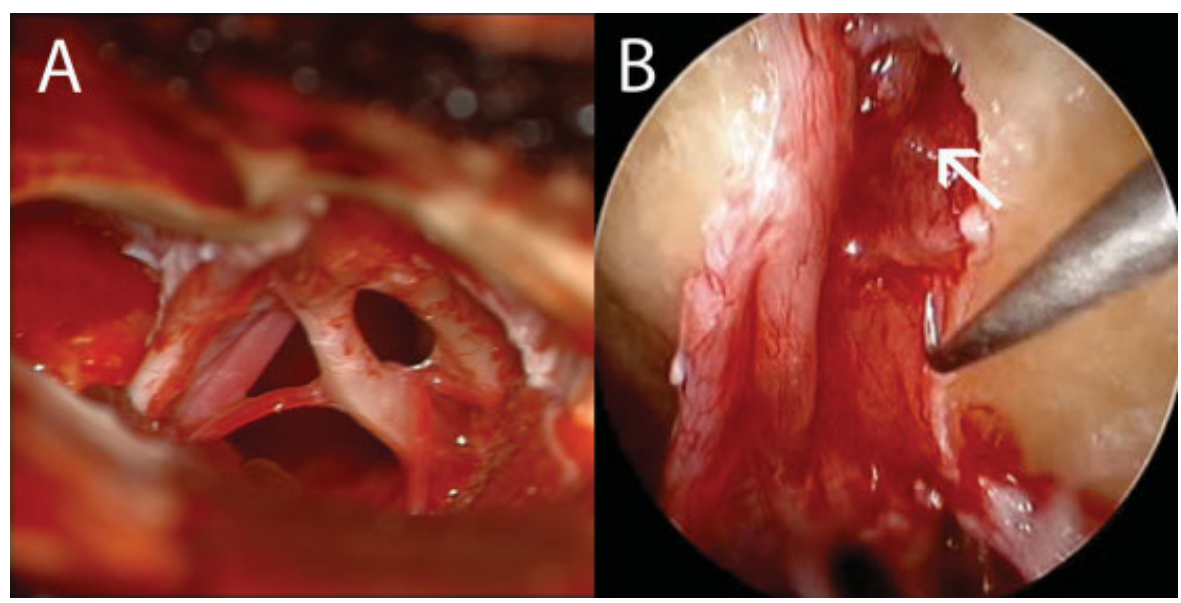

Fig. 3 Case A: After tumor resection with the microscope there appears to be no evidence of residual disease in the right IAC (A). When examined with the 30-degree endoscope, there is obvious residual tumor (arrow) that was not seen previously with microscopy (B). IAC, internal auditory canal. 


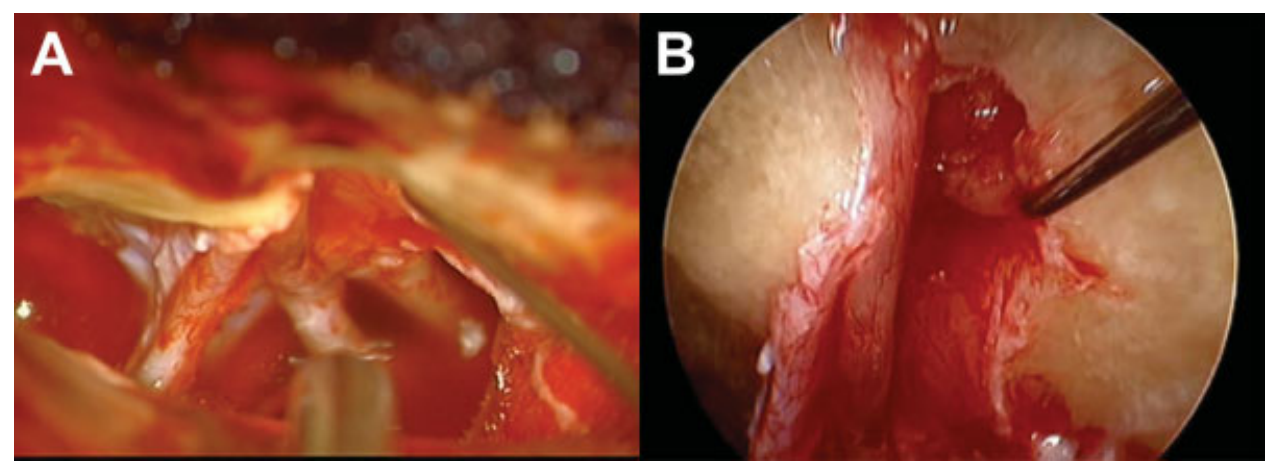

Fig. 4 Case A: Tumor dissection in the lateral IAC is conducted with the endoscope but is also under direct view of the microscope to not inadvertently injury surrounding structures. Notice the blind dissection that occurs under microscopy when the Mcelveen-Hitselberger neural dissector is placed into the IAC to remove residual tumor. On endoscopy, however, the facial and cochlear nerves are visualized so the end of the nerve dissector is not dissecting blindly, preventing unnecessary facial paresis, and hearing loss. IAC, internal auditory canal.

\section{Postoperative Results}

Postoperatively, the patient had a HB grade 3/6 facial paresis; this progressed to grade $5 / 6$ by the time of discharge and improved on last follow-up to HB 4/6. The patient was readmitted for ventriculitis 1 month after surgery and underwent shunt externalization; he was eventually successfully weaned from his VP shunt. Postoperative audiogram demonstrated a moderate improvement in hearing, with PTA of $47 \mathrm{~dB}$ and SDS of $60 \%$ (class B) ( - Fig. 1). Postoperative MRI at 3 months confirmed the absence of pathological enhancement or residual mass in the IAC (not shown).

\section{Patient B}

The patient is a 53-year-old male patient with a history of bilateral sensorineural hearing loss (SNHL) and a $5 \times 5 \mathrm{~mm}$ left intracanalicular acoustic neuroma ( - Fig. 6A). The patient

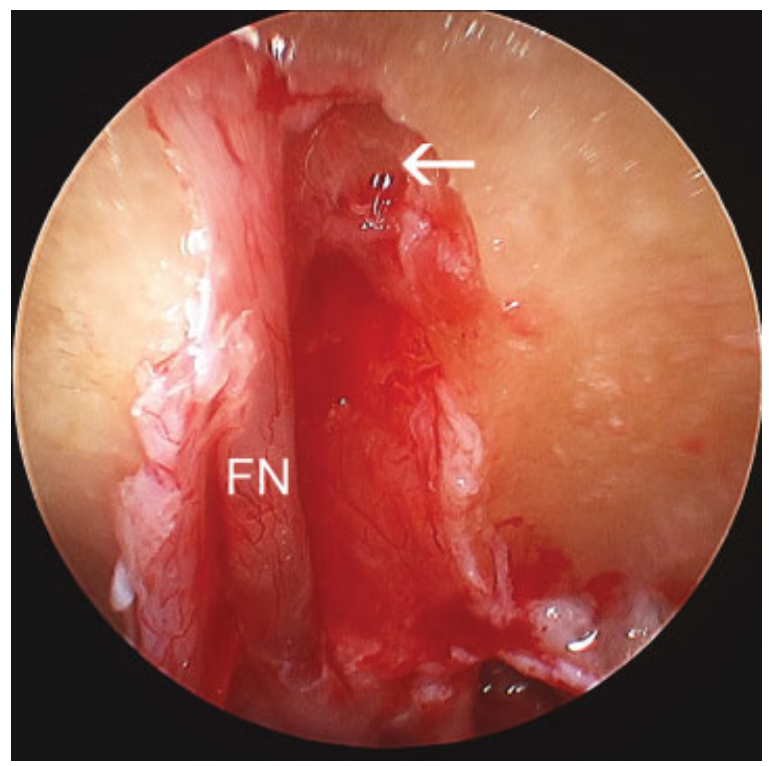

Fig. 5 Case A: After tumor resection with endoscopy in the lateral fundus, there is no residual tumor. With a 30-degree endoscope, the $\mathrm{FN}$ is depicted all the way to the fundus and transverse crest (arrow) can also be seen. FN, facial nerve. had a history of left-sided Bell palsy with recovered HB grade $1 / 6$ facial grade. The patient had a history of sudden hearing loss on the right that recovered with oral prednisone. He used hearing aids bilaterally. The remainder of his medical history was unremarkable.

MRI with high-resolution CISS imaging demonstrated a small tumor $(5 \mathrm{~mm})$ laterally impacted in the fundus of the left IAC. No fundal fluid was observable on T2-weighted imaging sequences (including the CISS sequence) ( - Fig. 6B). On audiologic testing the patient had $84 \%$ speech discrimination bilaterally; right ear PTA was $46 \mathrm{~dB}$ and left ear PTA was $50 \mathrm{~dB}$ (-Fig. 7). Despite the unfavorable location of the tumor, the patient wanted every chance on preserving his hearing so a MFC was utilized.

\section{Microsurgical Resection of Acoustic Neuroma}

The patient underwent a standard left-sided MFC for exposure of the IAC, as discussed above. After exposure of the posterior fossa dura and labyrinthine facial nerve, the dura was opened sharply and CSF drained from the exposed CPA. Fixed retraction of the temporal lobe was discontinued at this point, and the VS was resected using standard microsurgical dissection techniques. At the completion of the microsurgical portion of the procedure, no tumor could be seen in the IAC, although the lateral extent could not be visualized directly.

\section{Introduction of the Operative Endoscope}

The surgical endoscope was brought into the operative field in a similar manner as that described for case A, above. Upon completion of the microsurgical removal of tumor, cottonoids were placed on the surface of the temporal lobe dura and the surgical endoscope was introduced under direct observation through the operative microscope. After the fundus was visualized with an endoscope with a 70-degree viewing angle, it was felt that gross total tumor resection had been achieved with excellent visualization of the distal IAC, transverse crest, cochlear nerve, and facial nerve (-Fig. 8).

\section{Postoperative Results}

Postoperative facial nerve grade was HB grade1/6. Hearing was stable with a postoperative PTA of $51 \mathrm{~dB}$ and SDS slightly 


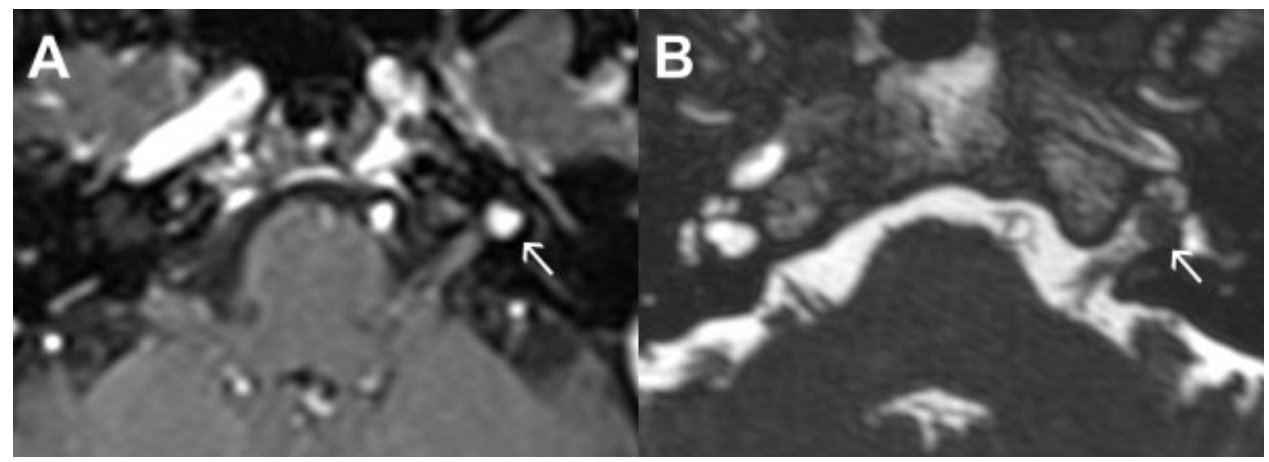

Fig. 6 Preoperative MRI for patient B. (A) T1-weighted MRI with contrast shows a left-sided lateral IAC hyperintense lesion at the fundus (white arrow). (B) T2-weighted MRI shows a filling defect in the lateral IAC and absence of fundal fluid lateral to the tumor (white arrow). IAC, internal auditory canal; MRI, magnetic resonance imaging.

diminished at 76\% (Fig. 7). Postoperative MRI at 3 months confirmed the absence of pathological enhancement or residual mass in the IAC (not shown).

\section{Discussion}

Use of the surgical endoscope has been proposed to help overcome the technical challenges of tumor dissection, especially in the lateral $\mathrm{IAC}^{8}$ In both the RS and MFC approaches, angled (30 and 70 degrees) endoscopes allow for up close high-definition visualization laterally toward the fundus, which is difficult with traditional microscopy. ${ }^{9}$

In the English literature, the majority of endoscopic-assisted VS resections are published for the RS approach. ${ }^{9-13}$ For this approach, the endoscope allows surgeons to not dissect blindly, which has often been the biggest disadvantage to the RS approach when tumors involve the lateral IAC. ${ }^{10}$ Even when endoscopic assisted, removal of tumor from the fundus is often still impossible due to obstruction by the posterior canal, common crus, and endolymphatic sac. ${ }^{14}$ In a cadaveric study by Pillai et al, only 70 to $80 \%$ of the posterior wall of the IAC could be drilled without injuring the labyrinth via a RS approach with image guidance. Visualization of the fundus was possible, however, with 30-degree endoscopes. ${ }^{14}$ In another study by Kumon et al, endoscopic-assisted microsurgical removal of VS through a RS approach only showed improved tumor removal in tumors extending medial to the mid portion of the IAC but not in tumors extending to the fundus. Interestingly, there was no difference in facial nerve function, hearing or tumor recurrence for endoscopic-assisted surgery in their cohort of 71 patients. ${ }^{15}$ When reported for MFC resections, the endoscope has only been used for inspection after tumor resection under the microscope. ${ }^{9}$ Only one article in the literature describes the endoscope to assisted dissection through the MFC approach, which was conducted on human cadavers. ${ }^{16}$

These two cases show the clear visual advantage of the endoscope for dissecting in the lateral IAC in MFC approaches. Dissection toward the fundus is limited by the neighboring cochlea and anterior semicircular canal as compared with the

\section{Case B Audiograms}

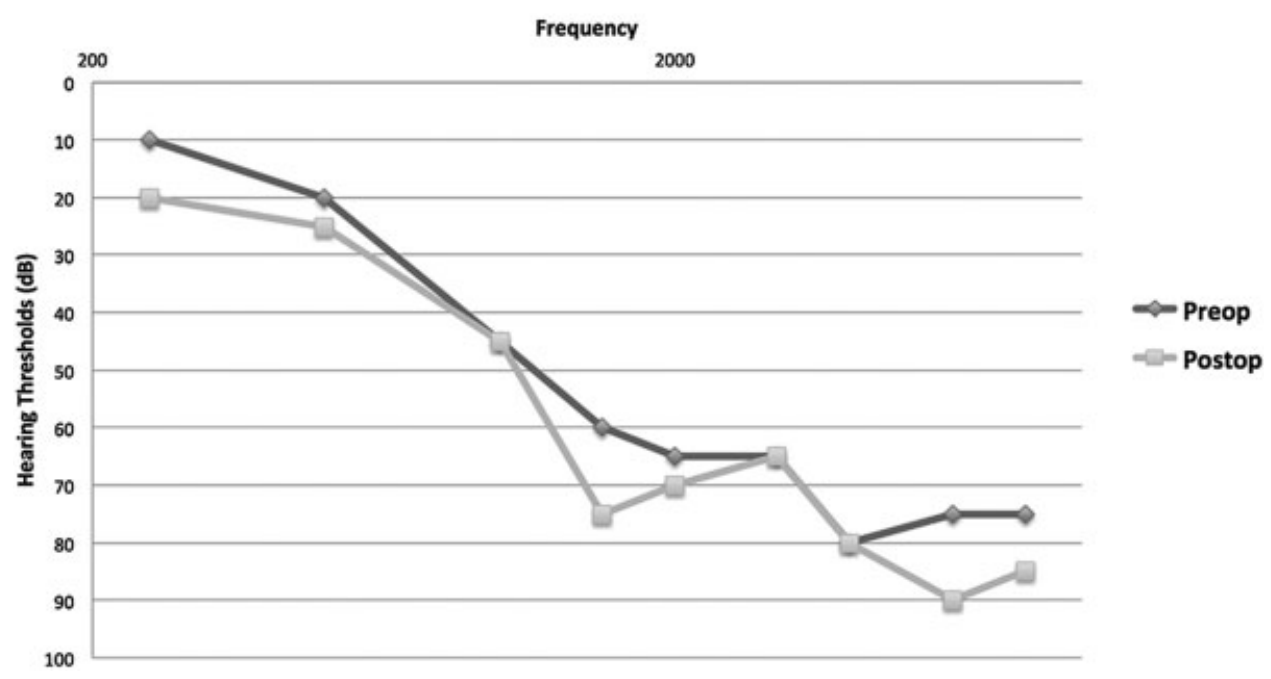

Fig. 7 Pre- and postoperative audiograms for patient B. Preoperative PTA is 48 (88\% SDS). Postoperative PTA is 51 (76\% SDS). PTA, pure tone audiometry; SDS, speech determination score. 


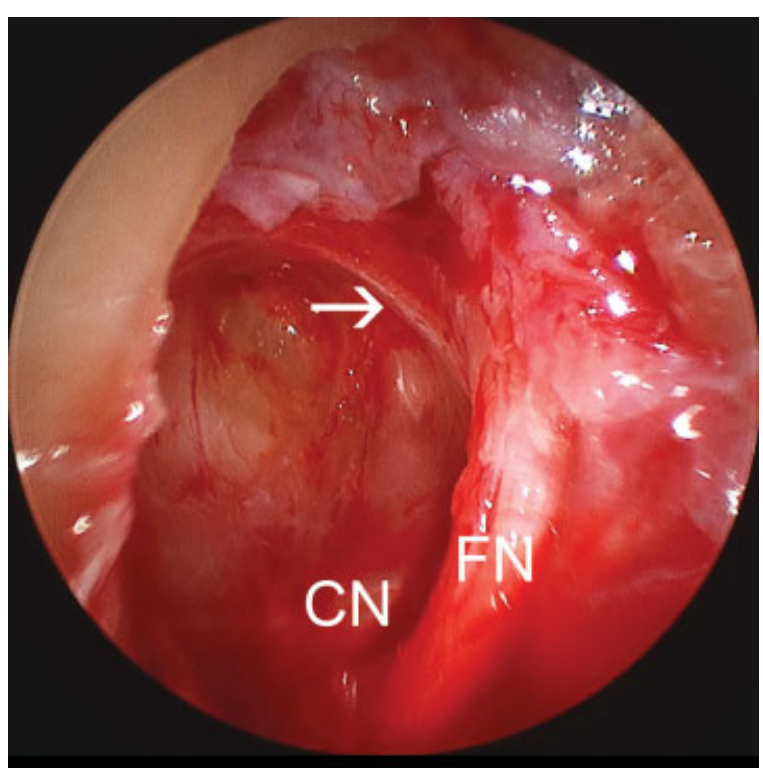

Fig. 8 Case B: Postendoscopic resection of the fundal VS, the transverse crest (arrow), FN, and CN is easily visualized with a 70degree endoscope. The integrity of both the nerves can be easily inspected during and postresection. CN, cochlear nerve; FN, facial nerve; VS, vestibular schwannoma.

270 degrees of dissection that is allowed toward the porus. For this reason, residual tumors can hide in the area vestibularis (area of the fundus where the superior and inferior vestibular nerves enter the vestibule), out of the direct line of sight of microscopy. With endoscopy, the most lateral extent of the fundus to include the transverse crest can be visualized.

One criticism of this technique is the potential risk of inadvertent direct injury to nearby structures with the endoscope itself when advancing it into position. ${ }^{17}$ This is especially true when trying to navigate an angled scope strictly using the endoscopic display. To minimize this we advance the endoscope under direct visualization of the microscope (as previously described when used in the RS approach). ${ }^{15}$ Once we are satisfied with the location of the endoscope by checking our view with both the microscope and the endoscopic display, the surgeon transitions attention to the endoscopic display for tumor resection, and the assistant maintains observation of the endoscope tip. The bulk of our tumor resection is still made under the microscope. Endoscopic assistance is only used when we suspect or cannot confirm residual disease in the lateral canal. Another potential pitfall of endoscopic resection is from heat produced by the light source of the endoscope, potentially damaging blood vessels (anterior inferior cerebellar artery, AICA) and nerves (facial nerve) that are in direct view. ${ }^{17}$ We postulate that this in fact may have contributed to facial weakness observed in patient $A$. The risk of facial nerve injury can be minimized by reducing the light source intensity (similar to that done for middle ear endoscopy) along with frequent irrigation, either manually with a syringe or with an Endoscrub (Medtronic, Minneapolis, Minnesota, United States).

\section{Conclusion}

We have demonstrated that the endoscope can improve visualization in the lateral IAC, where circumferential dissection is limited due to the proximity of the cochlea and vestibule. Our preliminary experience is encouraging in that we found utilization of the endoscope for identifying and resecting residual tumor not visualized under the microscope. We believe that the optical advantage of using the endoscope for tumor dissection may decrease the risk of injury to the cochlear nerve, thus improving hearing preservation. The potential for heat-related injury to the facial nerve, however, merits caution in the adoption of this technique. Given our early experience, we believe that prospective evaluation of the utility of surgical endoscopy in MFC is warranted to better define the appropriate role of this adjunct technology for microscopic resection of patients with intracanalicular tumors and serviceable hearing.

\section{Financial Disclosure}

There are no financial support or industry affiliations to disclose.

\section{References}

1 Slattery WH, Hoa M, Bonne N, et al. Middle fossa decompression for hearing preservation: a review of institutional results and indications. Otol Neurotol 2011;32(6):1017-1024

2 Kutz JW Jr, Scoresby T, Isaacson B, et al. Hearing preservation using the middle fossa approach for the treatment of vestibular schwannoma. Neurosurgery 2012;70(2):334-340, discussion 340-341

3 Sughrue ME, Yang I, Aranda D, Kane AJ, Parsa AT. Hearing preservation rates after microsurgical resection of vestibular schwannoma. J Clin Neurosci 2010;17(9):1126-1129

4 Wang AC, Chinn SB, Than KD, et al. Durability of hearing preservation after microsurgical treatment of vestibular schwannoma using the middle cranial fossa approach. J Neurosurg 2013;119(1): 131-138

5 Goddard JC, Schwartz MS, Friedman RA. Fundal fluid as a predictor of hearing preservation in the middle cranial fossa approach for vestibular schwannoma. Otol Neurotol 2010;31(7):1128-1134

6 Haberkamp TJ, Meyer GA, Fox M. Surgical exposure of the fundus of the internal auditory canal: anatomic limits of the middle fossa versus the retrosigmoid transcanal approach. Laryngoscope 1998; 108(8 Pt 1):1190-1194

7 Lateral Skull Base Surgery: The House Clinic Atlas. New York, NY: Thieme; 2012

8 Wackym PA, King WA, Meyer GA, Poe DS. Endoscopy in neurootologic surgery. Otolaryngol Clin North Am 2002;35(2):297-323

9 Wackym PA, King WA, Poe DS, et al. Adjunctive use of endoscopy during acoustic neuroma surgery. Laryngoscope 1999;109(8): 1193-1201

10 Göksu N, Bayazit Y, Kemaloğlu Y. Endoscopy of the posterior fossa and dissection of acoustic neuroma. J Neurosurg 1999;91(5): 776-780

11 Göksu N, Yilmaz M, Bayramoglu I, Aydil U, Bayazit YA. Evaluation of the results of endoscope-assisted acoustic neuroma surgery through posterior fossa approach. ORL J Otorhinolaryngol Relat Spec 2005;67(2):87-91

12 Miyazaki H, Deveze A, Magnan J. Neuro-otologic surgery through minimally invasive retrosigmoid approach: endoscope assisted 
microvascular decompression, vestibular neurotomy, and tumor removal. Laryngoscope 2005;115(9):1612-1617

13 Ebner FH, Roser F, Roder C, Tatagiba M, Schuhmann MU. Rigid, Variable-View Endoscope in Neurosurgery: First Intraoperative Experience. Surg Innov 2015;22(4):390-393

14 Pillai P, Sammet S, Ammirati M. Image-guided, endoscopic-assisted drilling and exposure of the whole length of the internal auditory canal and its fundus with preservation of the integrity of the labyrinth using a retrosigmoid approach: a laboratory investigation. Neurosurgery 2009;65(6, Suppl):53-59, discussion 59
15 Kumon Y, Kohno S, Ohue S, et al. Usefulness of endoscope-assisted microsurgery for removal of vestibular schwannomas. J Neurol Surg B Skull Base 2012;73(1):42-47

16 Low WK. Middle cranial fossa approach to the internal auditory Meatus:A Chinese temporal bone study. ORL J Otorhinolaryngol Relat Spec 1999;61(3):142-145

17 Gerganov VM, Giordano M, Herold C, Samii A, Samii M. An electrophysiological study on the safety of the endoscope-assisted microsurgical removal of vestibular schwannomas. Eur J Surg Oncol 2010;36(4):422-427 